\title{
DESIGN AND ANALYSIS OF DIFFERENT DIGITAL COMMUNICATION SYSTEMS AND DETERMINATION OF THE OPTIMUM SYSTEM
}

\author{
Sabyasachi Bhattacharyya ${ }^{1}$, Ragib Nasir Ahmed ${ }^{2}$ \\ Roushan Saikia ${ }^{3}$, Ripunjoy Sarma ${ }^{4}$, Kaustubh Bhattacharyya ${ }^{5}$ \\ DON BOSCO COLLEGE OF ENGINEERING AND TECHNOLOGY, Guwahati, INDIA \\ 'sabya005@gmail.com, ${ }^{2}$ ahmedragib@gmail.com, ${ }_{2}$ roushan.saikia@gmail.com, ${ }^{4}$ ripunjoy21@gmail.com \\ ${ }^{5}$ kaustubh.bhattacharyya@dbuniversity.ac.in
}

\begin{abstract}
For a specialized device or set-up in case of any practical communication related problem definition, a sound communication system consisting of the necessary integral parts is very crucial. As such, the design and an analysis of the various communication system is very critical as it directly effects the performance of the device and also reveals the inherent capacity of the system to produce the desired results. In this respect our topic for this paper is the design and analysis of different digital communication systems with a view to determine the most effective system considering all the parameters so that it can be used for important communication based problems and situations. In the next section we will mainly focus on the design processes of various systems, the theory involved, the simulation results, some special techniques of error correction and also the error performance of the systems.
\end{abstract}

Keywords- modulation, digital, communication systems, shift keying, error performance, SNR

$* * *$

\section{INTRODUCTION}

Digital communication systems are a very important and integral part of the modern day area of research in the field of Wireless Communication. This is because these digitized systems tend to exhibit better performance in terms of bandwidth, noise performance, power consumption and most importantly, for digital transmission, the error performance involved as compared to the traditional systems already existing. A key component in a typical digital system is the modulation technique and its corresponding demodulation involved. The various digital modulation schemes available are: Binary Amplitude Shift Keying (BASK), Binary Phase Shift Keying (BPSK), Binary Frequency Shift Keying (BFSK) and Quadrature Phase Shift Keying (QPSK) and some other modified versions of the above mentioned. With so many of these schemes available, the effective design of these systems and their performance analysis with respect to various parameters becomes very essential so as to derive the best communication system which would give the desired result with an optimized value for the different performance parameters i.e. the minimum possible error at the least possible signal to noise ratio (SNR) with minimum bandwidth for a noisy channel. In this discussion we will emphasize on the design and analysis of an optimized digital communication system for wireless applications. We will mainly concentrate on the schemes such as BASK, BFSK, BPSK and also a glimpse of the QPSK system, their design and waveforms with a view to determine the best scheme.
The flow of this report has been discussed as follows. The theoretical background of our work including details of different communication systems and the modulation and demodulation schemes involved have been explained in details in section I. A literature review of the work that has been carried out in this field and provided an idea behind some relevant papers has been discussed in section II. We have discussed about the work that we have practically done and in this regard we have discussed the design procedures that we have adopted for different systems and the essential components required for it and are given in section III. In the next section, we have presented the waveforms and various plots that we have obtained from the different designs and also a small analysis of the different systems have been provided. And finally in conclusion section we have discussed our inferences from the work that we have done.

\section{THEORITICAL BACKGROUND}

Over the years, the field of communication has been dominated by the traditional continuous wave modulation like amplitude modulation(AM),Frequency modulation(FM) and Phase modulation(PM), where one of the basic characteristics of a high frequency carrier signal is varied in accordance with the instantaneous changes in the low frequency message signal. But as we move to the digital domain, the task at hand becomes quite different as in this case, the data is in the form of bit streams( 0 's and 1's) and we have to apply the modulation or masking technique such that, in the transmitter 
end we represent the 1's as a one entity and 0's as a another. Further, we also have to take care that these masks are properly decoded at the receiver. However, it is seen that the digital modulation schemes that exist in practice have an essence of the traditional techniques too as they are directly associated to the amplitude, phase and frequency parameters of the carrier[1]. The most popular modulation schemes are:

\section{A. Amplitude shift Keying(ASK)}

Amplitude-shift keying (ASK) is a form of modulation that represents digital data as variations in the amplitude of a carrier wave. The simplest and most common form of ASK operates as a switch, using the presence of a carrier wave to indicate a binary ' 1 ' and its absence to indicate a binary ' 0 '. This type of modulation is called on-off keying, and is used at radio frequencies to transmit Morse code (referred to as continuous wave operation). Like AM, ASK is also linear and sensitive to atmospheric noise, distortions, propagation conditions on different routes in Public Switch Telephone Network (PSTN) etc. Both ASK modulation and demodulation processes are relatively inexpensive. The ASK technique is also commonly used to transmit digital data over optical fiber. For LED transmitters, binary 1 is represented by a short pulse of light and binary 0 by the absence of light. Laser transmitters normally have a fixed bias current that causes the device to emit a low light level. This low level represents binary 0 , while a higher-amplitude light wave represents binary 1. Generally, the performance of this modulation scheme is an average one and it is very much affected by noise. The demodulator, which is designed specifically for the symbol-set used by the modulator, determines the amplitude of the received signal and maps it back to the symbol it represents, thus recovering the original data, keeping frequency and phase of the carrier as constant[2].

\section{B. $\quad$ Frequency Shift keying(FSK)}

Frequency-shift keying (FSK) is a frequency modulation scheme in which digital informations are transmitted through discrete frequency changes of a carrier wave. The simplest FSK is binary FSK (BFSK). BFSK uses a pair of discrete frequencies to transmit binary ( $0 \mathrm{~s}$ and $1 \mathrm{~s})$ information. With this scheme, the ' 1 ' is called the mark frequency and the ' 0 ' is called the space frequency [2].

As far as demodulation of FSK signals are considered, it is a little difficult as compared to the phase modulated signals because the decision boundary is set at not such a suitable position in the constellation plot i.e at 45 degrees on the complex plane (1st quadrant). As a result, points lying close to the boundary stand the chance of producing erroneous results thus giving instability to the system [3].

\section{Phase Shift Keying(PSK)}

Phase-shift keying (PSK) is a modulation scheme that conveys data by changing, or modulating, the phase of a reference signal (the carrier wave). PSK uses a finite number of phases; each assigned a unique pattern of bits. Usually, each phase encodes an equal number of bits. Each pattern of bits forms the symbol that is represented by the particular phase. The demodulator, which is designed specifically for the symbol-set used by the modulator, determines the phase of the received signal and maps it back to the symbol it represents, thus recovering the original data. There are many versions of the PSK scheme defined as M-ary PSK. Here, we will be restricting our analysis into two forms:-

\section{a) BPSK(Binary Phase Shift Keying):-}

BPSK (also sometimes called Phase Reversal Keying (PRK), or 2PSK) is the simplest form of phase shift keying (PSK). It uses two phases which are separated by $180^{\circ}$ and so can also be termed 2-PSK. It does not matter where asexactly the constellation points are positioned. This modulation is the most robust of all the PSKs since it takes the highest level of noise or distortion to make the demodulator to make an incorrect decision. It is, however, only able to modulate at 1 bit/symbol (as seen in the figure) and so is unsuitable for high data rate. Here, $M$ value is 2. The general form for BPSK follows the equation:

$$
s_{n}(t)=\sqrt{\frac{2 E_{b}}{T_{b}}} \cos \left(2 \pi f_{c} t+\pi(1-n)\right), n=0,1 .
$$

This yields two phases, 0 and $\pi$. In the specific form, binary data is often conveyed with the following signals:

$$
\begin{aligned}
& s_{0}(t)=\sqrt{\frac{2 E_{b}}{T_{b}}} \cos \left(2 \pi f_{c} t+\pi\right)=-\sqrt{\frac{2 E_{b}}{T_{b}}} \cos \left(2 \pi f_{c} t\right) \\
& \text { (for binary "0") }
\end{aligned}
$$

$$
s_{1}(t)=\sqrt{\frac{2 E_{b}}{T_{b}}} \cos \left(2 \pi f_{c} t\right)
$$

(for binary "1")

where $f_{c}$ is the frequency of the carrier-wave.

\section{b) Quadrature phase Shift Keying(QPSK):-}

Sometimes this is known as quaternary PSK, quadriphase PSK, 4-PSK, or 4-QAM. (Although the root concepts of QPSK and 4-QAM are different, the resulting modulated radio waves are exactly the same.) QPSK uses four points on the 
constellation diagram, equispaced around a circle. With four phases, QPSK can encode two bits per symbol. i.e here the value of $M=4$. The implementation of QPSK is more general than that of BPSK and also indicates he implementation of higher-order PSK. Writing the symbols in the constellation diagram in terms of the sine and cosine waves used to transmit them:

$$
s_{n}(t)=\sqrt{\frac{2 E_{s}}{T_{s}}} \cos \left(2 \pi f_{c} t+(2 n-1) \frac{\pi}{4}\right), \quad n=1,2,3,4 .
$$

This yields the four phases $\pi / 4,3 \pi / 4,5 \pi / 4$ and $7 \pi / 4$ as needed.

In this discussion, we have emphasized more on the phase shifting scheme because it has been observed in general that as compared to the other shift keying techniques, the M-PSK scheme has lesser error probability due to the favorable constellation plots and decision boundaries. Also, the higher level PSK schemes like QPSK etc being di-bit system have higher data rates and thus can be used for high-speed transmission maintaining the bandwidth of the signals. Also, by keeping the same data rate as BPSK, we are able to reduce or half the effective bandwidth required. Thus from the above statements we are able to visualize that M-PSK systems have distinct advantages as compared to the other shift keying techniques and thus we have laid more emphasis on this scheme. This theoretical description has also been found to be true during the practical design of the systems [2].

\section{LITERATURE SURVEY}

The design and analysis of the digital modulation schemes being a very important topic of discussion in the field of Communication, a lot of study and work has been done over the years on this aspect of the design of a sound communication system. While performing our design and analysis, we have also gone through some of these proposed works to get an idea of the different approaches adopted:

\section{A Digital modulation Method for Terrestrial TV} Broadcasting is proposed by Mr.Saito and et all, where they have presented a very significant modulation technique i.e. OFDM system combined with Trellis Coded Modulation (TCM) for TV broadcasting and this system possesses the capability to transmit a large number of bits at a higher rate keeping the error performance almost constant. Also, the transmission has been tested under different receiving conditions and also carrier recovery is not required [4].Mr. Smithson discussed Digital Modulation Schemes and given an overview of each of the schemes in details. He suggests in his discussion that there is no such best communication system or scheme, it completely depends upon the parameters like channel characteristics, the error performance required, the BW availability, and also target cost for developing the hardware. Any system which optimally meets these requirements and with minimum trade-offs emerges as the final choice for a particular communication problem [5].

B Digital Modulation Classification Technique based on Power Moment Matrices, is presented by Mr.Alfred O.Hero and proposed a new approach based on the newly developed pattern recognition system to discriminate among the different M-ary PSK systems with respect to noise performance. Here, in this approach a matrix has been used for the process of eigen decomposition. The technique has been applied to greyscale images. Here, the noise is being applied to the images and then it is being decomposed to eigen values. It is then being fed to the different systems and analysis is done as to which system can restore the image nearest to its desirable form [6].

\section{PRESENT WORK}

As mentioned, in the previous section, we have designed a sound communication system which would optimally meet the requirements related to noise, error performance, bandwidth requirement, hardware complexity and the SNR requirement etc. So, for this, we have carried out the designing of the various systems and done their analysis as well. Besides, we are further designing some more relevant systems and are trying to make a comparative study between all of them to find the optimum one. The channel that we have used is the AWGN channel. We have successfully implemented the design for three of the schemes and the details are given as follows:-

\section{B. BASK System:}

Design Process:

- A bit stream of data is accepted at input.

- It is converted to a pulse shaped digital signal.

- Modulate the pulse with a sinusoidal carrier.

- The channel response is added to it at certain range of value of SNR.

- Coherent demodulation is done to separate out carrier from message signal.

- A threshold value check logic is used to determine the output pulse for the receiver.

- The input and output bit streams are compared.

\section{BPSK System:}

Design Process:

- Bit stream is accepted at input.

- Converted to a pulse signal.

- Converted to a polar pulse signal.

- Modulated with a proper sinusoid. 
- Signal is passed over AWGN channel at optimum value of SNR.

- Coherent Demodulation separates the carrier from the message.

- Over the bit duration, the energy associated with the symbols is calculated.

- Using a Threshold Check Logic, we determine which symbol corresponds to a bit 0 and which one gives bit 1.

- The input and output bit streams are compared.

\section{BFSK System:}

Design Process :

- Bit stream is accepted at the input terminals.

- Converted to a pulse signal.

- Encoding is done or we can say a control switch operation is taking place.

- Modulation is done for the parted data with two different frequencies one high representing high and other giving low.

- Both the modulated outputs are aggregated into one.

- The signal is passed through AWGN channel.

- At the receiver, the similar steps such as coherent demodulation, symbol representation by a signed value and also threshold checking is done.

- The input and output pulse are compared.

Note: - For optimization of BW, we are trying to develop the di-bit QPSK system. But have not yet succeeded in fully completing this scheme and so we are not disclosing the design process in this discussion. However, we have got satisfactory results for the baseband version of QPSK. So, we have included the results obtained for those in the next section.

\section{RESULTS AND DISCUSSIONS}

In this section, we have discussed the output waveforms that we have obtained for the different systems that we have designed till date and also we have made an analysis of the performance of the different schemes.

\section{E. BASK System:}

Having designed the BASK system, we have got the following results and analysed the performance of the system which are shown in Figure 1:

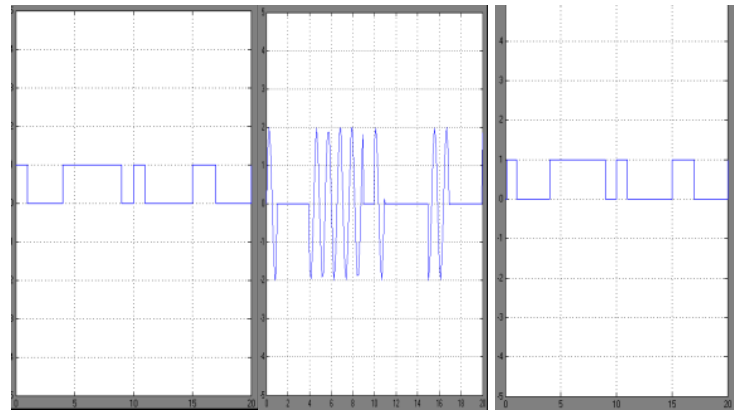

$\begin{array}{lll}\text { Figure 1.1 } & \text { Figure 1.2 } & \text { Figure1.3 }\end{array}$

Figure 1: 1.1: Input Signal

1.2: Modulated signal

1.3: Output signal

From Figure 1 we have seen that the input and output pulses are the same. The modulation is done with sinusoidal carrier of frequency $1 \mathrm{KHz}$. The demerit, regarding this system is that at low values of SNR, the performance is not that good and it is difficult to operate at very high values of SNR in practical situations. This happens mainly because of the fact that the presence and absence of signal gives the output and so while decoding a little bit of noise can change a bit 0 to a 1 .

\section{F. BPSK System:}

Having designed the BPSK system, we have got the following results and analysed the performance of the system which are shown in Figure 2:

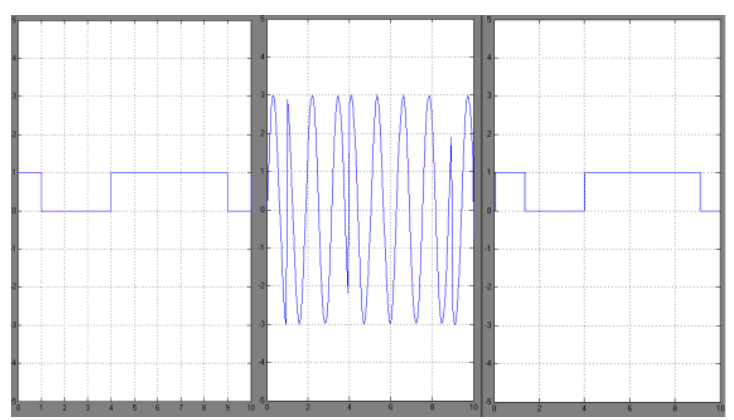

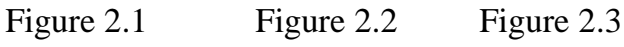

Figure 2: 2.1: Input Signal

2.2: Modulated Signal

2.3: Output Signal

1. The input and output pulses as shown are the same.

2. The bipolar pulse is generated to get two different signed values of energy of signal thus accounting for a proper decision boundary.

3. A $10 \mathrm{kHz}$ carrier signal is used for the modulation. 
4. The good part about the system is that at low SNR, the demodulation is proper as the decision boundary is very definite depending on the sign assigned to each symbol.

5. However, it is a single-bit system and so BW required is higher.

6.For phase modulated signal, we have least error probability.

\section{G. BFSK System:}

Having designed the BFSK system, we have got the following results and analysed the performance of the system which are shown in Figure 3:

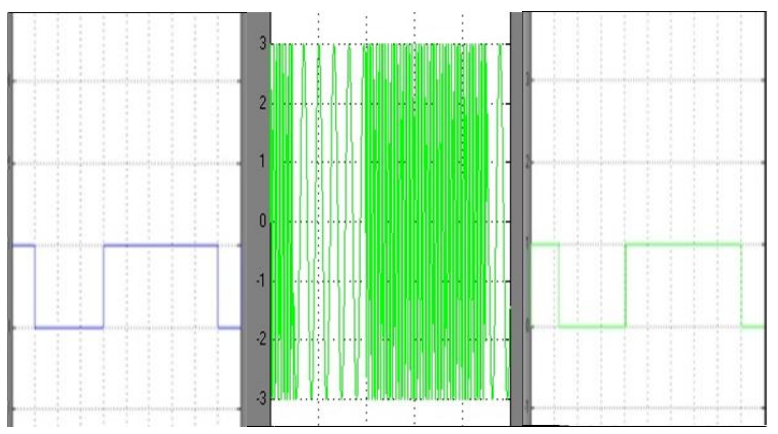

Figure3.1

Figure 3.2

Figure3.3

Figure 3: 3.1: Input Signal

\section{2: Modulated signal}

\section{3: Output signal}

The BFSK system is an average performance system. It has better performance than the BASK system. But there is more chance of error probability than the BPSK system. Because it has the two extreme points lying in $\mathrm{y}$-axis and $\mathrm{x}$-axis line. So, when the symbol is somewhere around diagonal position chances of error are more for BFSK as compared to BPSK. Thus the system is less stable. It is also a single bit system and there is high $\mathrm{BW}$ required.

\section{H. QPSK Baseband Modulation:}

Having designed the QPSK Baseband system, we have got the following results and analysed the performance of the system which are shown in Figure 4:

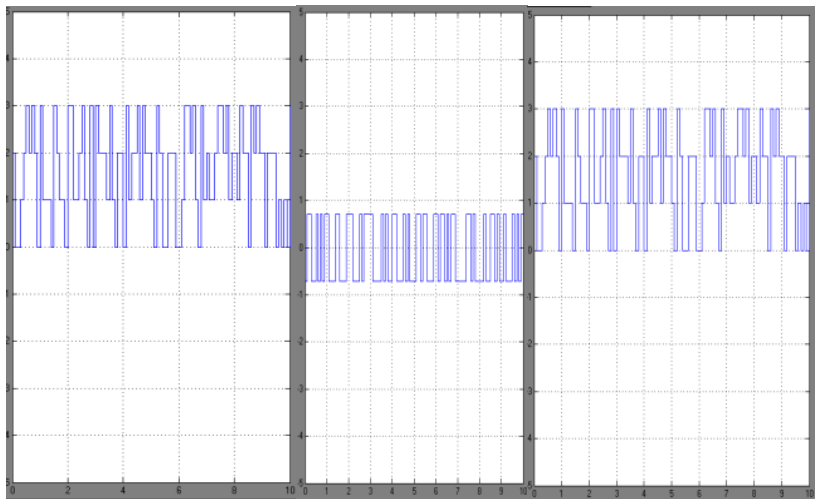

$\begin{array}{lll}\text { Figure } 4.1 & \text { Figure4.2 } & \text { Figure } 4.3\end{array}$

Figure4: 4.1: Input Signal 4.2: Modulated Signal 4.3: Output signal

\section{- Power spectral Density plot:}

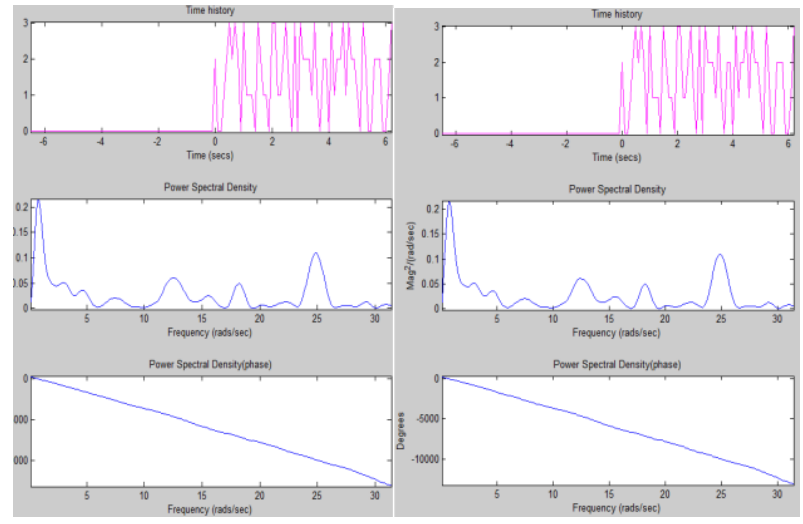

Figure5.1

Figure5.2

Figure5: Power Spectral Density plot

\section{- Constellation Plots:}

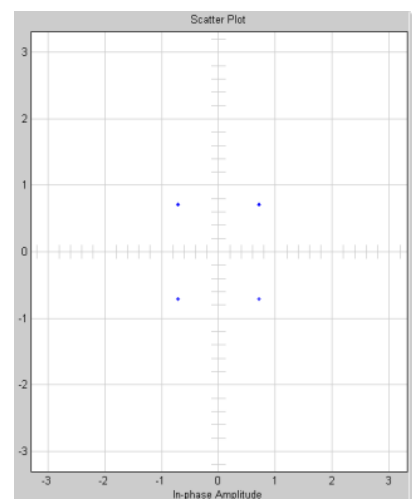

Figure 6.1

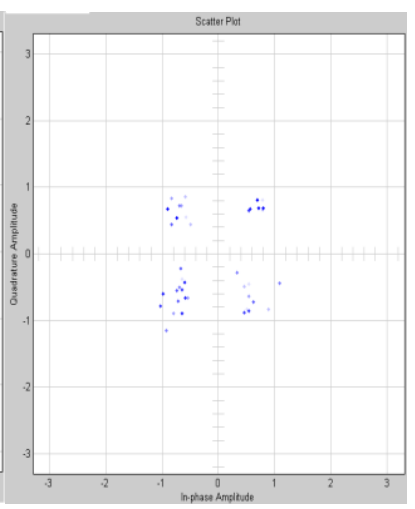

Figure 6.2
Figure6: 6.1: Coordinate of energy level 6.2: Scatter Plot 
From Figure 5 and 6 it is seen that at baseband level analysis itself, the QPSK scheme shows very impressive performance. Thus, it can easily be predicted what it would perform with a carrier modulation added to it. With this view in mind we are trying out to design the passband model for this communication system. But we have not yet succeeded in it.

\section{CONCLUSIONS}

In this section, we would like to draw certain conclusions related to our work till date. As already discussed, we have designed and analyzed BASK, BPSK, BFSK and partially the QPSK system also. Having done so we can say that the Phase Shift Keying systems have more stability and show better performance in terms of error chances, noise effect and also in terms of bit rate and bandwidth. This is largely due to the fact that the BPSK system have suitable constellation diagram and decision boundary which results in proper decoding. Also QPSK being a di bit system helps increasing the bit rate by almost twice that of BPSK..

\section{ACKNOWLEDGMENTS}

At the very outset, we would like to thank Kaustubh Bhattacharyya sir, Assistant Professor, Department of Electronics and Communication Engineering, Don Bosco College of Engineering and Technology for guiding us in our work.. We would also like to thank all the teachers of our department and all the technical and non-technical staffs for their valuable inputs to our work. We would also like to thank some of our friends for their constant motivation for preparing this report.

\section{REFERENCES}

[1]. B.P. Lathi, Passband Modulation Techniques. Modern Digital and Analog Communication

[2]. Simon Haykin, Digital Passband Transmission "Communication Systems", Fourth Edition, Wiley India Edition, pages (344-458)

[3]. Basab. B.Purkayastha and Kandarpa Kumar Sarma. A Digital Phase Locked Loop for Nakagami-m fading Channels using QPSK modulation schemes. $2^{\text {nd }}$ IEEE National Conference on Computational Intelligence and Signal Processing, pages 1-20, 2011.

[4]. Masafumi Saito, Shigeki Moriyama, Osamu Yamada, “A Digital Modulation Method for Terrestrial Digital TV Broadcasting Using Trellis Coded OFDM and its Performance", International Conference on Electronics and Communication Technology, Guwahati, pages 1-5

June 2012.

[5]. Geoff Smithson, "Introduction to Digital Modulation Schemes", Proceedings of the ISITA2010, Taichum, Taiwan, pp. 928-933, Oct., 2010

[6]. Alfred O. Hero III, Hafez Hadinejad-Mahram, "Digital Modulation Classification Using Power Moment Matrices", IEEE transactions on Circuits and Systems-II: Express Briefs, vol. 52, no. 3, Mar, 2005. 